\title{
Contents of Volume 1
}

Preface

List of Tables

Notes on Institutional Exhibits

Accreditation in Higher Education

Abbreviations and Definitions

v

ix

xi

xiii

xvii

\section{Higher Education in the United States}

1. The Evolution of Higher Education 3

2. Higher Education for a New Millennium $\quad 7$

3. The Structure of Higher Education 9

4. Undergraduate Education 17

5. Graduate and Professional Education 23

6. Government and Higher Education 27

7. Foreign Students in the United States 33

8. College Is Possible $\quad 57$

\section{Professional Education in the United States}

$\begin{array}{lr}\text { Architecture } & 61 \\ \text { Art and Design } & 63 \\ \text { Business Administration } & 65 \\ \text { City and Regional Planning } & 69 \\ \text { Computing Science } & 70 \\ \text { Construction Education } & 72 \\ \text { Counseling } & 74 \\ \text { Culinary } & 76 \\ \text { Dance } & 77 \\ \text { Dietetics } & 78 \\ \text { Engineering and Engineering Technology } & \\ \text { Engineering } & 81 \\ \text { Engineering Technology } & 88 \\ \text { Family and Consumer Sciences } & 94 \\ \quad \text { Marriage \& Family Therapy } & 95 \\ \text { Forestry } & 96 \\ \text { Funeral Service } & 98 \\ \text { Health Professions } & \\ \text { Acupuncture and Oriental Medicine } & 99 \\ \text { Allied Health } & 100 \\ \text { Chiropractic Education } & 119 \\ \text { Clinical Laboratory Science } & 120 \\ \text { Dentistry } & 124 \\ \text { Health Services Administration } & 128 \\ \text { Medicine } & 129 \\ \text { Nuclear Medicine Technology } & 131 \\ \text { Nursing } & 133 \\ \quad \text { Nurse Anesthesia } & 143\end{array}$

$\begin{array}{ll}\text { Health Professions (cont.) } & \\ \text { Occupational Therapy } & 144 \\ \text { Optometry } & 147 \\ \text { Osteopathic Medicine } & 148 \\ \text { Pharmacy } & 149 \\ \text { Physical Therapy } & 150 \\ \text { Podiatry } & 154 \\ \text { Public Health } & 154 \\ \text { Rehabilitation Counseling } & 156 \\ \text { Speech-Language Pathology } & \\ \quad \text { and Audiology } & 158 \\ \text { Interior Design } & 161 \\ \text { Journalism and Mass Communications } & 163 \\ \text { Landscape Architecture } & 164 \\ \text { Law } & 166 \\ \text { Librarianship } & 168 \\ \text { Music } & 169 \\ \text { Psychology } & 175 \\ \text { Public Affairs and Administration } & 178 \\ \text { Recreation and Park Planning } & 179 \\ \text { Social Work } & 181 \\ \text { Teacher and School Service } & \\ \text { Personnel Education } & 186 \\ \text { Theater } & 191 \\ \text { Theology and Rabbinical/Talmudic } & \\ \quad \text { Education } & 193 \\ \text { Theology } & 196 \\ \text { Rabbinical and Talmudic Education } & 197 \\ \text { Veterinary Medicine } & \end{array}$

\section{Universities and Colleges: Institutional Exhibits}

$\begin{array}{llll}\text { Alabama } & 201 & \text { Delaware } & 414 \\ \text { Alaska } & 230 & \text { District of Columbia } & 419 \\ \text { Arizona } & 235 & \text { Florida } & 436 \\ \text { Arkansas } & 247 & \text { Georgia } & 475 \\ \text { California } & 264 & \text { Hawaii } & 514 \\ \text { Colorado } & 367 & \text { Idaho } & 521 \\ \text { Connecticut } & 389 & \text { Illinois } & 529\end{array}$




$\begin{array}{llll}\text { Indiana } & 601 & \text { Maine } & 757 \\ \text { Iowa } & 648 & \text { Maryland } & 774 \\ \text { Kansas } & 678 & \text { Massachusetts } & 800 \\ \text { Kentucky } & 703 & \text { Michigan } & 868 \\ \text { Louisiana } & 732 & \text { Minnesota } & 917\end{array}$

\section{Contents of Volume 2}

Preface

List of Tables

Notes on Institutional Exhibits

v

ix

Accreditation in Higher Education

xi

Abbreviations and Definitions

xiii

xvii

\section{Universities and Colleges: Institutional Exhibits (continued)}

Mississippi
Missouri
Montana
Nebraska
Nevada
New Hampshire
New Jersey
New Mexico
New York
North Carolina
North Dakota
Ohio
Oklahoma
Oregon
Pennsylvania

\section{Appendices}

1. An Academic Costume Code and An Academic Ceremony Guide

2. Tables of Earned Doctorates and Master's Degrees Conferred by American Universities and Colleges, 1869-1997

3. ROTC Units: Army, Navy, and Air Force

Puerto Rico
Rhode Island
South Carolina
South Dakota
Tennessee
Texas
Utah
Vermont
Virginia
Washington
West Virginia
Wisconsin
Wyoming
U.S. Trust Territories

4. Summary Data by State for Institutions Appearing in Part III

5. American Council on Education

\section{Indices}

Institutional Index 